\title{
Effective Bounds for Very Ample Line Bundles
}

\author{
Jean-Pierre Demailly \\ Institut Fourier, Université de Grenoble I
}

Summary. Let $L$ be an ample line bundle on a non singular projective $n$-fold $X$. It is first shown that $2 K_{X}+m L$ is very ample for $m \geq 2+\left(\begin{array}{c}3 n+1 \\ n\end{array}\right)$. The proof developes an original idea of Y.T. Siu and is based on a combination of the Riemann-Roch theorem together with an improved Noetherian induction technique for the Nadel multiplier ideal sheaves. In the second part, an effective version of the big Matsusaka theorem is obtained, refining an earlier version of Y.T. Siu: there is an explicit polynomial bound $m_{0}=m_{0}\left(L^{n}, L^{n-1} \cdot K_{X}\right)$ of degree $\leq n 3^{n}$ in the arguments, such that $m L$ is very ample for $m \geq m_{0}$. The refinement is obtained through a new sharp upper bound for the dualizing sheaves of algebraic varieties embedded in projective space.

\section{Introduction}

In the last six or seven years, considerable progress has been achieved in the understanding of adjoint linear systems $\left|K_{X}+m L\right|$ associated with an ample line bundle $L$ on a smooth projective manifold $X$. When $X$ is a surface, I. Reider [Rei88] obtained a quasi-optimal criterion for the global generation and very ampleness of $K_{X}+L$, showing in particular that $K_{X}+3 L$ is always generated by global sections and $K_{X}+4 L$ very ample. Around the same period, T. Fujita [Fuj87] raised the following interesting conjecture.

(0.1) Conjecture (Fujita). Let $X$ be a smooth projective $n$-fold over $\mathbb{C}$ and let $L$ be an ample line bundle on $X$. Then $K_{X}+(n+1) L$ is generated by global sections and $K_{X}+(n+2) L$ is very ample.

One of the first results proved in dimension $n \geq 3$ is the very ampleness of $2 K_{X}+12 n^{n} L$, using an analytic method based on the solution of a Monge-Ampère equation (see [Dem93]). Slightly later, J. Kollár [Kol93] obtained an effective version of the base point free theorem, while a major step was made in small dimension by L. Ein and R. Lazarsfeld [EL93], with the solution of the global generation part of Fujita's conjecture for $n=3$. Other related works are [EL92], [Fuj94], [EKL94], [Ein94] (see also [Laz93] and [Dem94] for survey expositions). Recently, Y.T. Siu [Siu94a] introduced a simple algebraic method for proving the very ampleness of $2 K_{X}+m L$. His method is based on a combination of the Riemann-Roch formula with the Kawamata-Viehweg vanishing theorem, in the generalized form given by A. Nadel [Nad89]. Our first goal is to develope a more efficient Noetherian induction process for the Nadel multiplier sheaves associated with singular hermitian metrics, along the lines of Siu's method. The new induction process is simpler and allows us to refine further Siu's original bounds. In the sequel the intersection numbers of $L$ over $d$-dimensional subvarieties $Y \subset X$ are denoted 


$$
L^{d} \cdot Y=\int_{Y} c_{1}(L)^{d}
$$

We say that $L$ is numerically effective (nef for short) if $L \cdot C \geq 0$ for every algebraic curve $C \subset X$. By [Dem90], $L$ is nef if and only if for each $\varepsilon>0$ there is a hermitian metric $h_{\varepsilon}$ on $L$ of curvature $\Theta_{h_{\varepsilon}}(L) \geq-\varepsilon \omega$, where $\omega$ is a given Kähler form on $X$.

(0.2) Theorem. Let $X$ be a smooth projective $n$-fold and let $L$ be an ample line bundle over $X$. Then

a) $2 K_{X}+m L$ is very ample for $m \geq 2+\left(\begin{array}{c}3 n+1 \\ n\end{array}\right)$;

b) $2 K_{X}+L$ generates simultaneous jets of order $s_{1}, \ldots, s_{p}$ at arbitrary points $x_{1}, \ldots, x_{p} \in X$ provided that the intersection numbers $L^{d} \cdot Y$ of $L$ over all $d$-dimensional algebraic subsets $Y$ of $X$ satisfy

$$
L^{d} \cdot Y>\frac{2^{d-1}}{\lfloor n / d\rfloor^{d}} \sum_{1 \leq j \leq p}\left(\begin{array}{c}
(n+1)\left(4 n+2 s_{j}+1\right)-2 \\
n
\end{array}\right), \quad 1 \leq d \leq n .
$$

c) $m\left(K_{X}+(n+2) L\right)$ is very ample for $m \geq\left(\begin{array}{c}3 n+1 \\ n\end{array}\right)-2 n$.

All results still hold true by adding any nef line bundle $G$ to the line bundles under consideration.

Our method of proof is sharp enough to yield as a by-product the well-known result that $K_{X}+(n+1) L$ is numerically effective if $L$ is ample (a result originally proved as a consequence of Mori theory). A basic problem would be to find an analogue of Th. $(0.2 \mathrm{a}, \mathrm{b})$ with $K_{X}$ in place of $2 K_{X}$. For the global generation question, the answer has been settled in the affirmative recently by U. Angehrn and Y.T. Siu [AS94], who showed that $K_{X}+\frac{1}{2}\left(n^{2}+n+2\right) L$ is always generated by global sections; their method is again based on Nadel's vanishing theorem, using a different idea for the construction of the required singular hermitian metrics. The result of AngehrnSiu implies that $K_{X}+2 n\left(K_{X}+\frac{1}{2}\left(n^{2}+n+2\right) L\right)$ is very ample for $n \geq 2$ (by the elementary observation that $K_{X}+2 n F$ is always very ample if $F$ is ample and generated by sections); the bound obtained in $(0.2 \mathrm{c})$ can then be improved into $m \geq n^{3}$. In a related paper [Siu94b], Y.T. Siu obtains a variant of $(0.2 \mathrm{~b})$ in which the numerical condition for $L^{d} \cdot Y$ is replaced by $\left(L^{d} \cdot Y\right)^{1 / d}>2 n \sum_{1 \leq j \leq p}\left(\begin{array}{c}3 n+2 s_{j}-3 \\ n\end{array}\right)+2 p n$; this bound, which has a rather involved proof, is sharper than ours for $d \leq O(\ln (n))$ but weaker for larger values of $d$. At the time these lines are written, it seems to be unknown whether there is a bound $m_{0}(n)$ depending only on the dimension such that $K_{X}+m L$ is very ample for $m$ larger than $m_{0}(n)$. Also it seems to be unknown whether polynomial bounds $m_{0}(n)$ exist for $2 K_{X}+m L$ (the bound given by $(0.2 \mathrm{a}$ ) is of the order of magnitude of $(27 / 4)^{n}$ and seems to be the best presently known).

Another important question is to find effective bounds $m_{0}$ such that $m L$ becomes very ample for $m \geq m_{0}$. From a theoretical point of view, this problem is solved by Matsusaka [Mat72] and Kollár-Matsusaka [KoM83]. Their result states that there is a bound $m_{0}=m_{0}\left(n, L^{n}, L^{n-1} \cdot K_{X}\right)$ depending only on the dimension and on the first two coefficients $L^{n}$ and $L^{n-1} \cdot K_{X}$ in the Hilbert polynomial of $L$. Unfortunately, the original proof does not tell much on the actual dependence of 
$m_{0}$ in terms of these coefficients. In a ground-breaking paper [Siu93], Y.T. Siu introduced new techniques leading to effective bounds for $m_{0}$. The published version of [Siu93] incorporates an induction argument which we developed in collaboration with the author after the preprint version circulated, enabling us to obtain much better final estimates. Our goal in the last sections $\S 3,4$ is to present a further substantial refinement of this method. The main point is that a crucial technical lemma used in [Siu93] to deal with dualizing sheaves can be made optimal by using a different idea based on the Ohsawa-Takegoshi $L^{2}$ extension theorem [OT87].

(0.3) Theorem. Let $H$ be a very ample line bundle on a projective algebraic manifold $X$, and let $Y \subset X$ be a p-dimensional irreducible algebraic subvariety. Denote by $\omega_{Y}$ the $L^{2}$ dualizing sheaf of $Y$. If $\delta=H^{p} \cdot Y$ is the degree of $Y$ with respect to $H$, the sheaf $\mathcal{H o m}\left(\omega_{Y}, \mathcal{O}_{Y}((\delta-p-2) H)\right)$ has a nontrivial section.

Using this sharp "upper estimate" on dualizing sheaves and some other refinements of the inductive method explained in [Siu93], we obtain the following improved bounds.

(0.4) Theorem. If $L$ is an ample line bundle on a projective $n$-fold $X$, then $m L$ is very ample for

$$
m \geq m_{0}=C_{n}\left(L^{n}\right)^{3^{n-2}}\left(n+2+\frac{L^{n-1} \cdot K_{X}}{L^{n}}\right)^{3^{n-2}(n / 2+3 / 4)+1 / 4}
$$

where $C_{n}$ depends only on $n$, e.g.,

$$
C_{n}=(2 n)^{\left(3^{n-1}-1\right) / 2}\left(\left(\begin{array}{c}
3 n+1 \\
n
\end{array}\right)-2 n\right)^{3^{n-2}(n / 2+3 / 4)+1 / 4} .
$$

The bound (0.4) turns out to be essentially optimal for $n=2$ (apart from a small multiplicative constant), as was shown recently by Fernández del Busto [FdB94] by means of Reider's theorem and an example of Gang Xiao. Our bound is probably not optimal for $n \geq 3$, and we strongly believe that there should exist an optimal bound of the form $C_{n}\left(L^{n}\right)^{a_{n}}\left(n+2+L^{n-1} \cdot K_{X} / L^{n}\right)^{b_{n}}$, involving exponents $a_{n}, b_{n}$ of the order of magnitude of $n$ or $n^{2}$ instead of $n 3^{n}$.

\section{Nadel's Vanishing Theorem}

We recall here briefly a few basic ideas developed in [Dem90,93], which will be equally useful in this paper. Let $X$ be a projective algebraic manifold equipped with a Kähler metric $\omega$, and let $F$ be a holomorphic line bundle over $X$. We assume that $F$ is equipped with a (possibly singular) hermitian metric $h$. In each open set $U$ where $F_{\lceil U} \simeq U \times \mathbb{C}$ is trivial, the metric $h$ is given by a weight $\varphi$ such that $\|\xi\|_{h}=|\tau(\xi)| \mathrm{e}^{-\varphi(x)}$ for all $\xi \in F_{x}$, where $\tau: F_{\mid U} \rightarrow \mathbb{C}$ is the trivialization map. If $\varphi$ is supposed to be locally integrable on $U$, the curvature form of $F$ can be defined to 
be the closed $(1,1)$-current $\Theta_{h}(F)=\frac{i}{\pi} \partial \bar{\partial} \varphi$. Here, we will only consider the case of nonnegative curvature currents $\Theta_{h}(F) \geq 0$, i.e., we suppose that the weights $\varphi$ are plurisubharmonic. Following Nadel [Nad89], we associate to $\varphi$ the ideal sheaf

$$
\mathcal{I}(\varphi)=\left\{f \in \mathcal{O}_{X, x} ; \exists W \ni x, \int_{W}|f|^{2} \mathrm{e}^{-2 \varphi} d V_{\omega}<+\infty\right\}
$$

where $d V_{\omega}=\omega^{n} / n$ ! is the Kähler volume form and $W$ is an arbitrary open neighborhood of $x$. Of course, $\mathcal{I}(\varphi)$ does not depend on the choice of the trivialization, and thus we get a global ideal sheaf $\mathcal{I}(h)$ on $X$ depending only on $h$. By [Nad89] and [Dem93], $\mathcal{I}(h)$ is a coherent ideal sheaf in $\mathcal{O}_{X}$, and we have the following fundamental vanishing theorem.

(1.2) Nadel vanishing theorem. Assume that $\Theta_{h}(F) \geq \varepsilon \omega$ for some $\varepsilon>0$. Then

$$
H^{q}\left(X, \mathcal{O}\left(K_{X}+F\right) \otimes \mathcal{I}(h)\right)=0 \quad \text { for all } q \geq 1 \text {. }
$$

The proof is a straightforward consequence of the Bochner-Kodaira-Nakano identity ([AN54], [Nak55]) and of Hörmander's $L^{2}$ estimates for the $\bar{\partial}$ operator (see [Hör65], [AV65], [Nad89], [Dem93]). In the present paper, we only need "algebraic" metrics $h$ of the form

$$
\|\xi\|_{h}^{2}=\frac{|\tau(\xi)|^{2}}{\left(\sum_{1 \leq j \leq N}\left|\tau^{\mu}\left(\sigma_{j}(x)\right)\right|^{2}\right)^{1 / \mu}}
$$

where $\sigma_{1}, \ldots, \sigma_{N} \in H^{0}(X, \mu F)$ are non zero algebraic sections of $\mu F=F^{\otimes \mu}$, and $\tau^{\mu}$ is the local trivialization of $F^{\otimes \mu}$ induced by a local trivialization $\tau$ of $F$. The corresponding weight is

$$
\varphi=\frac{1}{2 \mu} \log \left(\sum_{1 \leq j \leq N}\left|\tau^{\mu}\left(\sigma_{j}(x)\right)\right|^{2}\right) .
$$

In this case, (1.2) is equivalent to the Kawamata-Viehweg vanishing ([Kaw82], [Vie82]), and the proof can be reduced to the usual Kodaira vanishing theorem by purely algebraic means. Now, recall that the Lelong number of a plurisubharmonic function $\varphi$ at a point $x$ is $\nu(\varphi, x)=\lim _{r \rightarrow 0} \sup _{B(x, r)} \varphi / \log r$. In the special case (1.4) under consideration, we simply have

$$
\nu(\varphi, x)=\frac{1}{m} \min _{1 \leq j \leq N} \operatorname{ord}_{x}\left(\sigma_{j}\right)
$$

where $\operatorname{ord}_{x}\left(\sigma_{j}\right)$ is the vanishing order of $\sigma_{j}$ at $x$.

(1.5) Corollary. Let $(X, \omega), F, h$ and $\varphi$ be as in (1.2) and let $x_{1}, \ldots, x_{N}$ be isolated points in the zero variety $V(\mathcal{I}(\varphi))$. Then there is a surjective map

$$
H^{0}\left(X, K_{X}+F\right) \longrightarrow \bigoplus_{1 \leq j \leq N} \mathcal{O}\left(K_{X}+F\right)_{x_{j}} \otimes\left(\mathcal{O}_{X} / \mathcal{I}(h)\right)_{x_{j}}
$$


In particular, if $\nu\left(\varphi, x_{j}\right) \geq n+s_{j}$, then $H^{0}\left(X, K_{X}+F\right)$ generates simultaneously all jets of order $s_{j}$ at $x_{j}$.

Proof. Consider the long exact sequence of cohomology associated to the short exact sequence $0 \rightarrow \mathcal{I}(\varphi) \rightarrow \mathcal{O}_{X} \rightarrow \mathcal{O}_{X} / \mathcal{I}(\varphi) \rightarrow 0$ twisted by $\mathcal{O}\left(K_{X}+F\right)$, and apply Th. (1.2) to obtain the vanishing of the first $H^{1}$ group. The asserted surjectivity property follows. The last statement follows from the fact that $\nu(\varphi) \geq n+s$ implies $\mathcal{I}(h)_{x} \subset \mathfrak{m}_{x}^{s+1}$. Indeed, we then have

$$
\varphi(z) \leq(n+s) \log |z-x|+O(1), \quad \mathrm{e}^{-\varphi(z)} \geq c|z-x|^{-(n+s)}, \quad c>0,
$$

as is obvious in the "algebraic case" (in general, the inequality follows from the standard logarithmic convexity property of plurisubharmonic functions).

(1.6) Remark. As is well known, Corollary (1.6) can be proved by a direct application of Hörmander's $L^{2}$ estimates, namely by solving a $\bar{\partial}$-equation $\bar{\partial} u=\sum \bar{\partial}\left(\psi_{j} P_{j}\right)$ for forms of type $(n, 1)$, where $P_{j}$ is a finite holomorphic Taylor expansion achieving the desired jet at $x_{j}$, and where $\psi_{j}$ is a cut-off function with support in a neighborhood of $x_{j}$. In this way, we see that Cor. (1.6) still holds if we only have $\Theta_{h}(F) \geq 0$ and $\Theta_{h}(F) \geq \varepsilon \omega$ in a neighborhood of each $x_{j}$.

\section{Some Results Around the Fujita Conjecture}

This section is devoted to a proof of various results related to the Fujita conjecture. The main ideas occuring here are inspired by a very recent work of Y.T. Siu [Siu94a]. His method, which is algebraic in nature and quite elementary, consists in a combination of the Riemann-Roch formula together with Nadel's vanishing theorem (in fact, only the algebraic case is needed, thus the original Kawamata-Viehweg vanishing theorem would be sufficient). In the sequel, $X$ denotes a projective algebraic $n$-dimensional manifold. The first observation is the following well-known consequence of the Riemann-Roch formula.

(2.1) Special case of Riemann-Roch. Let $\mathcal{J} \subset \mathcal{O}_{X}$ be a coherent ideal sheaf on $X$ such that the subscheme $Y=V(\mathcal{J})$ has dimension $d$ (with possibly some lower dimensional components). Let $[Y]=\sum \lambda_{j}\left[Y_{j}\right]$ be the effective algebraic cycle of dimension $d$ associated to the $d$ dimensional components of $Y$ (taking into account multiplicities $\lambda_{j}$ given by the ideal $\left.\mathcal{J}\right)$. Then for any line bundle $F$, the Euler characteristic

$$
\chi\left(Y, \mathcal{O}(F+m L)_{\uparrow Y}\right)=\chi\left(X, \mathcal{O}(F+m L) \otimes \mathcal{O}_{X} / \mathcal{J}\right)
$$

is a polynomial $P(m)$ of degree $d$ and leading coefficient $L^{d} \cdot[Y] / d$ !

The second fact is an elementary lemma about numerical polynomials (polynomials with rational coefficients, mapping $\mathbb{Z}$ into $\mathbb{Z}$ ). 
(2.2) Lemma. Let $P(m)$ be a numerical polynomial of degree $d>0$ and leading coefficient $a_{d} / d$ !, $a_{d} \in \mathbb{Z}, a_{d}>0$. Suppose that $P(m) \geq 0$ for $m \geq m_{0}$. Then

a) For every integer $N \geq 0$, there exists $m \in\left[m_{0}, m_{0}+N d\right]$ such that $P(m) \geq N$.

b) For every $k \in \mathbb{N}$, there exists $m \in\left[m_{0}, m_{0}+k d\right]$ such that $P(m) \geq a_{d} k^{d} / 2^{d-1}$.

c) For every integer $N \geq 2 d^{2}$, there exists $m \in\left[m_{0}, m_{0}+N\right]$ such that $P(m) \geq N$.

Proof. a) Each of the $N$ equations $P(m)=0, P(m)=1, \ldots, P(m)=N-1$ has at most $d$ roots, so there must be an integer $m \in\left[m_{0}, m_{0}+d N\right]$ which is not a root of these.

b) By Newton's formula for iterated differences $\Delta P(m)=P(m+1)-P(m)$, we get

$$
\Delta^{d} P(m)=\sum_{1 \leq j \leq d}(-1)^{j}\left(\begin{array}{l}
d \\
j
\end{array}\right) P(m+d-j)=a_{d}, \quad \forall m \in \mathbb{Z} .
$$

Hence if $j \in\{0,2,4, \ldots, 2\lfloor d / 2\rfloor\} \subset[0, d]$ is the even integer achieving the maximum of $P\left(m_{0}+d-j\right)$ over this finite set, we find

$$
2^{d-1} P\left(m_{0}+d-j\right)=\left(\left(\begin{array}{l}
d \\
0
\end{array}\right)+\left(\begin{array}{l}
d \\
2
\end{array}\right)+\ldots\right) P\left(m_{0}+d-j\right) \geq a_{d},
$$

whence the existence of an integer $m \in\left[m_{0}, m_{0}+d\right]$ with $P(m) \geq a_{d} / 2^{d-1}$. The case $k=1$ is thus proved. In general, we apply the above case to the polynomial $Q(m)=P\left(k m-(k-1) m_{0}\right)$, which has leading coefficient $a_{d} k^{d} / d$ !

c) If $d=1$, part a) already yields the result. If $d=2$, a look at the parabola shows that

$$
\max _{m \in\left[m_{0}, m_{0}+N\right]} P(m) \geq \begin{cases}a_{2} N^{2} / 8 & \text { if } N \text { is even, } \\ a_{2}\left(N^{2}-1\right) / 8 & \text { if } N \text { is odd }\end{cases}
$$

thus $\max _{m \in\left[m_{0}, m_{0}+N\right]} P(m) \geq N$ whenever $N \geq 8$. If $d \geq 3$, we apply b) with $k$ equal to the smallest integer such that $k^{d} / 2^{d-1} \geq N$, i.e. $k=\left\lceil 2(N / 2)^{1 / d}\right\rceil$, where $\lceil x\rceil \in \mathbb{Z}$ denotes the round-up of $x \in \mathbb{R}$. Then $k d \leq\left(2(N / 2)^{1 / d}+1\right) d \leq N$ whenever $N \geq 2 d^{2}$, as a short computation shows.

We now apply Nadel's vanishing theorem pretty much in the same way as Siu [Siu94a], but with substantial simplifications in the technique and improvements in the bounds. Our method yields simultaneously a simple proof of the following basic result.

(2.3) Theorem. If $L$ is an ample line bundle over a projective $n$-fold $X$, then $K_{X}+(n+1) L$ is nef.

By using Mori theory and the base point free theorem ([Mor82], [Kaw84]), one can even show that $K_{X}+(n+1) L$ is semiample, i.e., there exists a positive integer $m$ such that $m\left(K_{X}+(n+1) L\right)$ is generated by sections (see [Kaw85] and [Fuj87]). The proof rests on the observation that $n+1$ is the maximal length of extremal rays of smooth projective $n$-folds. Our proof of (2.3) is different and will be given simultaneously with the proof of Th. (2.4) below. 
(2.4) Theorem. Let $L$ be an ample line bundle and let $G$ be a nef line bundle on a projective $n$-fold $X$. Then the following properties hold.

a) $2 K_{X}+m L+G$ generates simultaneous jets of order $s_{1}, \ldots, s_{p} \in \mathbb{N}$ at arbitrary points $x_{1}, \ldots, x_{p} \in X$, i.e., there is a surjective map

$$
H^{0}\left(X, 2 K_{X}+m L+G\right) \longrightarrow \bigoplus_{1 \leq j \leq p} \mathcal{O}\left(2 K_{X}+m L+G\right) \otimes \mathcal{O}_{X, x_{j}} / \mathfrak{m}_{X, x_{j}}^{s_{j}+1}
$$

provided that $m \geq 2+\sum_{1 \leq j \leq p}\left(\begin{array}{c}3 n+2 s_{j}-1 \\ n\end{array}\right)$.

In particular $2 K_{X}+m L+G$ is very ample for $m \geq 2+\left(\begin{array}{c}3 n+1 \\ n\end{array}\right)$.

b) $2 K_{X}+(n+1) L+G$ generates simultaneous jets of order $s_{1}, \ldots, s_{p}$ at arbitrary points $x_{1}, \ldots, x_{p} \in X$ provided that the intersection numbers $L^{d} \cdot Y$ of $L$ over all d-dimensional algebraic subsets $Y$ of $X$ satisfy

$$
L^{d} \cdot Y>\frac{2^{d-1}}{\lfloor n / d\rfloor^{d}} \sum_{1 \leq j \leq p}\left(\begin{array}{c}
3 n+2 s_{j}-1 \\
n
\end{array}\right) .
$$

Proof. The proofs of $(2.3)$ and $(2.4 \mathrm{a}, \mathrm{b})$ go along the same lines, so we deal with them simultaneously (in the case of $(2.3)$, we simply agree that $\left\{x_{1}, \ldots, x_{p}\right\}=\emptyset$ ). The idea is to find an integer (or rational number) $m_{0}$ and a singular hermitian metric $h_{0}$ on $K_{X}+m_{0} L$ with strictly positive curvature current $\Theta_{h_{0}} \geq \varepsilon \omega$, such that $V\left(\mathcal{I}\left(h_{0}\right)\right)$ is 0 -dimensional and the weight $\varphi_{0}$ of $h_{0}$ satisfies $\nu\left(\varphi_{0}, x_{j}\right) \geq n+s_{j}$ for all $j$. As $L$ and $G$ are nef, $\left(m-m_{0}\right) L+G$ has for all $m \geq m_{0}$ a metric $h^{\prime}$ whose curvature $\Theta_{h^{\prime}}$ has arbitrary small negative part (see [Dem90]), e.g., $\Theta_{h^{\prime}} \geq-\frac{\varepsilon}{2} \omega$. Then $\Theta_{h_{0}}+\Theta_{h^{\prime}} \geq \frac{\varepsilon}{2} \omega$ is again positive definite. An application of Cor (1.5) to $F=K_{X}+m L+G=\left(K_{X}+m_{0} L\right)+\left(\left(m-m_{0}\right) L+G\right)$ equipped with the metric $h_{0} \otimes h^{\prime}$ implies the existence of the desired sections in $K_{X}+F=2 K_{X}+m L+G$ for $m \geq m_{0}$.

Let us fix an embedding $\Phi_{|\mu L|}: X \rightarrow \mathbb{P}^{N}, \mu \gg 0$, given by sections $\lambda_{0}, \ldots, \lambda_{N} \in$ $H^{0}(X, \mu L)$, and let $h_{L}$ be the associated metric on $L$ of positive definite curvature form $\omega=\Theta(L)$. In order to obtain the desired metric $h_{0}$ on $K_{X}+m_{0} L$, we fix $a \in \mathbb{N}^{\star}$ and use a double induction process to construct singular metrics $\left(h_{k, \nu}\right)_{\nu \geq 1}$ on $a K_{X}+b_{k} L$ for a non increasing sequence of positive integers $b_{1} \geq b_{2} \geq \ldots \geq$ $b_{k} \geq \ldots$ Such a sequence much be stationary and $m_{0}$ will just be the stationary $\operatorname{limit} m_{0}=\lim b_{k} / a$. The metrics $h_{k, \nu}$ are taken to satisfy the following properties:

a) $h_{k, \nu}$ is an algebraic metric of the form

$$
\|\xi\|_{h_{k, \nu}}^{2}=\frac{\left|\tau_{k}(\xi)\right|^{2}}{\left(\sum_{1 \leq i \leq \nu, 0 \leq j \leq N}\left|\tau_{k}^{(a+1) \mu}\left(\sigma_{i}^{a \mu} \cdot \lambda_{j}^{(a+1) b_{k}-a m_{i}}\right)\right|^{2}\right)^{1 /(a+1) \mu}}
$$

defined by sections $\sigma_{i} \in H^{0}\left(X,(a+1) K_{X}+m_{i} L\right), m_{i}<\frac{a+1}{a} b_{k}, 1 \leq i \leq \nu$, where $\xi \mapsto \tau_{k}(\xi)$ is an arbitrary local trivialization of $a K_{X}+b_{k} L$; note that $\sigma_{i}^{a \mu} \cdot \lambda_{j}^{(a+1) b_{k}-a m_{i}}$ is a section of 


$$
a \mu\left((a+1) K_{X}+m_{i} L\right)+\left((a+1) b_{k}-a m_{i}\right) \mu L=(a+1) \mu\left(a K_{X}+b_{k} L\right) .
$$

B) $\operatorname{ord}_{x_{j}}\left(\sigma_{i}\right) \geq(a+1)\left(n+s_{j}\right)$ for all $i, j$;

$\gamma) \mathcal{I}\left(h_{k, \nu+1}\right) \supset \mathcal{I}\left(h_{k, \nu}\right)$ and $\mathcal{I}\left(h_{k, \nu+1}\right) \neq \mathcal{I}\left(h_{k, \nu}\right)$ whenever the zero variety $V\left(\mathcal{I}\left(h_{k, \nu}\right)\right)$ has positive dimension.

The weight $\varphi_{k, \nu}=\frac{1}{2(a+1) \mu} \log \sum\left|\tau_{k}^{(a+1) \mu}\left(\sigma_{i}^{a \mu} \cdot \lambda_{j}^{(a+1) b_{k}-a m_{i}}\right)\right|^{2}$ of $h_{k, \nu}$ is plurisubharmonic and the condition $m_{i}<\frac{a+1}{a} b_{k}$ implies $(a+1) b_{k}-a m_{i} \geq 1$, thus the difference $\varphi_{k, \nu}-\frac{1}{2(a+1) \mu} \log \sum\left|\tau\left(\lambda_{j}\right)\right|^{2}$ is also plurisubharmonic. Hence $\Theta_{h_{k, \nu}}\left(a K_{X}+b_{k} L\right)=$ $\frac{\mathrm{i}}{\pi} \partial \bar{\partial} \varphi_{k, \nu} \geq \frac{1}{(a+1)} \omega$. Moreover, condition $\left.\beta\right)$ clearly implies $\nu\left(\varphi_{k, \nu}, x_{j}\right) \geq a\left(n+s_{j}\right)$. Finally, condition $\gamma$ ) combined with the strong Noetherian property of coherent sheaves ensures that the sequence $\left(h_{k, \nu}\right)_{\nu \geq 1}$ will finally produce a zero dimensional subscheme $V\left(\mathcal{I}\left(h_{k, \nu}\right)\right)$. We agree that the sequence $\left(h_{k, \nu}\right)_{\nu \geq 1}$ stops at this point, and we denote by $h_{k}=h_{k, \nu}$ the final metric, such that $\operatorname{dim} V\left(\mathcal{I}\left(h_{k}\right)\right)=0$.

For $k=1$, it is clear that the desired metrics $\left(h_{1, \nu}\right)_{\nu \geq 1}$ exist if $b_{1}$ is taken large enough (so large, say, that $(a+1) K_{X}+\left(b_{1}-1\right) L$ generates jets of order $(a+1)\left(n+\max s_{j}\right)$ at every point; then the sections $\sigma_{1}, \ldots, \sigma_{\nu}$ can be chosen with $\left.m_{1}=\ldots=m_{\nu}=b_{1}-1\right)$. Suppose that the metrics $\left(h_{k, \nu}\right)_{\nu \geq 1}$ and $h_{k}$ have been constructed and let us proceed with the construction of $\left(h_{k+1, \nu}\right)_{\nu \geq 1}$. We do this again by induction on $\nu$, assuming that $h_{k+1, \nu}$ is already constructed and that $\operatorname{dim} V\left(\mathcal{I}\left(h_{k+1, \nu}\right)\right)>0$. We start in fact the induction with $\nu=0$, and agree in this case that $\mathcal{I}\left(h_{k+1,0}\right)=0$ (this would correspond to an infinite metric of weight identically equal to $-\infty)$. By Nadel's vanishing theorem applied to $F_{m}=a K_{X}+$ $m L=\left(a K_{X}+b_{k} L\right)+\left(m-b_{k}\right) L$ with the metric $h_{k} \otimes\left(h_{L}\right)^{\otimes m-b_{k}}$, we get

$$
H^{q}\left(X, \mathcal{O}\left((a+1) K_{X}+m L\right) \otimes \mathcal{I}\left(h_{k}\right)\right)=0 \quad \text { for } q \geq 1, m \geq b_{k} .
$$

As $V\left(\mathcal{I}\left(h_{k}\right)\right)$ is 0-dimensional, the sheaf $\mathcal{O}_{X} / \mathcal{I}\left(h_{k}\right)$ is a skyscraper sheaf, and the exact sequence $0 \rightarrow \mathcal{I}\left(h_{k}\right) \rightarrow \mathcal{O}_{X} \rightarrow \mathcal{O}_{X} / \mathcal{I}\left(h_{k}\right) \rightarrow 0$ twisted with the invertible sheaf $\mathcal{O}\left((a+1) K_{X}+m L\right)$ shows that

$$
H^{q}\left(X, \mathcal{O}\left((a+1) K_{X}+m L\right)\right)=0 \quad \text { for } q \geq 1, m \geq b_{k} .
$$

Similarly, we find

$$
H^{q}\left(X, \mathcal{O}\left((a+1) K_{X}+m L\right) \otimes \mathcal{I}\left(h_{k+1, \nu}\right)\right)=0 \quad \text { for } q \geq 1, m \geq b_{k+1}
$$

(also true for $\nu=0$, since $\mathcal{I}\left(h_{k+1,0}\right)=0$ ), and when $m \geq \max \left(b_{k}, b_{k+1}\right)=b_{k}$, the exact sequence $0 \rightarrow \mathcal{I}\left(h_{k+1, \nu}\right) \rightarrow \mathcal{O}_{X} \rightarrow \mathcal{O}_{X} / \mathcal{I}\left(h_{k+1}, \nu\right) \rightarrow 0$ implies

$$
H^{q}\left(X, \mathcal{O}\left((a+1) K_{X}+m L\right) \otimes \mathcal{O}_{X} / \mathcal{I}\left(h_{k+1, \nu}\right)\right)=0 \quad \text { for } q \geq 1, m \geq b_{k} .
$$

In particular, since the $H^{1}$ group vanishes, every section $u^{\prime}$ of $(a+1) K_{X}+m L$ on the subscheme $V\left(\mathcal{I}\left(h_{k+1, \nu}\right)\right)$ has an extension $u$ to $X$. Fix a basis $u_{1}^{\prime}, \ldots, u_{N}^{\prime}$ of the sections on $V\left(\mathcal{I}\left(h_{k+1, \nu}\right)\right)$ and take arbitrary extensions $u_{1}, \ldots, u_{N}$ to $X$. Look at the linear map assigning the collection of jets of order $(a+1)\left(n+s_{j}\right)-1$ at all points $x_{j}$

$$
u=\sum_{1 \leq j \leq N} a_{j} u_{j} \longmapsto \bigoplus J_{x_{j}}^{(a+1)\left(n+s_{j}\right)-1}(u)
$$


Since the rank of the bundle of $s$-jets is $\left(\begin{array}{c}n+s \\ n\end{array}\right)$, the target space has dimension

$$
\delta=\sum_{1 \leq j \leq p}\left(\begin{array}{c}
n+(a+1)\left(n+s_{j}\right)-1 \\
n
\end{array}\right) .
$$

In order to get a section $\sigma_{\nu+1}=u$ satisfying condition $\beta$ ) with non trivial restriction $\sigma_{\nu+1}^{\prime}$ to $V\left(\mathcal{I}\left(h_{k+1, \nu}\right)\right)$, we need at least $N=\delta+1$ independent sections $u_{1}^{\prime}, \ldots, u_{N}^{\prime}$. This condition is achieved by applying Lemma (2.2) to the numerical polynomial

$$
\begin{aligned}
P(m) & =\chi\left(X, \mathcal{O}\left((a+1) K_{X}+m L\right) \otimes \mathcal{O}_{X} / \mathcal{I}\left(h_{k+1, \nu}\right)\right) \\
& =h^{0}\left(X, \mathcal{O}\left((a+1) K_{X}+m L\right) \otimes \mathcal{O}_{X} / \mathcal{I}\left(h_{k+1, \nu}\right)\right) \geq 0, \quad m \geq b_{k} .
\end{aligned}
$$

The polynomial $P$ has degree $d=\operatorname{dim} V\left(\mathcal{I}\left(h_{k+1, \nu}\right)\right)>0$. We get the existence of an integer $m \in\left[b_{k}, b_{k}+\eta\right]$ such that $N=P(m) \geq \delta+1$ with some explicit integer $\eta \in \mathbb{N}$ (for instance $\eta=n(\delta+1)$ always works by $(2.2 \mathrm{a})$, but we will also use the other possibilities to find an optimal choice in each case). Then we find a section $\sigma_{\nu+1} \in H^{0}\left(X,(a+1) K_{X}+m L\right)$ with non trivial restriction $\sigma_{\nu+1}^{\prime}$ to $V\left(\mathcal{I}\left(h_{k+1, \nu}\right)\right)$, vanishing at order $\geq(a+1)\left(n+s_{j}\right)$ at each point $x_{j}$. We just set $m_{\nu+1}=m$, and the condition $m_{\nu+1}<\frac{a+1}{a} b_{k+1}$ is satisfied if $b_{k}+\eta<\frac{a+1}{a} b_{k+1}$. This shows that we can take inductively

$$
b_{k+1}=\left\lfloor\frac{a}{a+1}\left(b_{k}+\eta\right)\right\rfloor+1 .
$$

By definition, $h_{k+1, \nu+1} \leq h_{k+1, \nu}$, hence $\mathcal{I}\left(h_{k+1, \nu+1}\right) \supset \mathcal{I}\left(h_{k+1, \nu}\right)$. We necessarily have $\mathcal{I}\left(h_{k+1, \nu+1}\right) \neq \mathcal{I}\left(h_{k+1, \nu}\right)$, for $\mathcal{I}\left(h_{k+1, \nu+1}\right)$ contains the ideal sheaf associated with the zero divisor of $\sigma_{\nu+1}$, whilst $\sigma_{\nu+1}$ does not vanish identically on $V\left(\mathcal{I}\left(h_{k+1, \nu}\right)\right)$. Now, an easy computation shows that the iteration of $b_{k+1}=$ $\left\lfloor\frac{a}{a+1}\left(b_{k}+\eta\right)\right\rfloor+1$ stops at $b_{k}=a(\eta+1)+1$ for any large initial value $b_{1}$. In this way, we obtain a metric $h_{\infty}$ of positive definite curvature on $a K_{X}+(a(\eta+1)+1) L$, with $\operatorname{dim} V\left(\mathcal{I}\left(h_{\infty}\right)\right)=0$ and $\nu\left(\varphi_{\infty}, x_{j}\right) \geq a\left(n+s_{j}\right)$ at each point $x_{j}$.

Proof of (2.3). In this case, the set $\left\{x_{j}\right\}$ is taken to be empty, thus $\delta=0$. By (2.2 a), the condition $P(m) \geq 1$ is achieved for some $m \in\left[b_{k}, b_{k}+n\right]$ and we can take $\eta=n$. As $\mu L$ is very ample, there exists on $\mu L$ a metric with an isolated logarithmic pole of Lelong number 1 at any given point $x_{0}$ (e.g., the algebraic metric defined with all sections of $\mu L$ vanishing at $\left.x_{0}\right)$. Hence

$$
F_{a}^{\prime}=a K_{X}+(a(n+1)+1) L+n \mu L
$$

has a metric $h_{a}^{\prime}$ such that $V\left(\mathcal{I}\left(h_{a}^{\prime}\right)\right)$ is zero dimensional and contains $\left\{x_{0}\right\}$. By Cor (1.5), we conclude that

$$
K_{X}+F_{a}^{\prime}=(a+1) K_{X}+(a(n+1)+1+n \mu) L
$$

is generated by sections, in particular $K_{X}+\frac{a(n+1)+1+n \mu}{a+1} L$ is nef. As $a$ tends to $+\infty$, we infer that $K_{X}+(n+1) L$ is nef.

Proof of (2.4 a). Here, the choice $a=1$ is sufficient for our purposes. Then 


$$
\delta=\sum_{1 \leq j \leq p}\left(\begin{array}{c}
3 n+2 s_{j}-1 \\
n
\end{array}\right) .
$$

If $\left\{x_{j}\right\} \neq \emptyset$, we have $\delta+1 \geq\left(\begin{array}{c}3 n-1 \\ n\end{array}\right)+1 \geq 2 n^{2}$ for $n \geq 2$. Lemma (2.2c) shows that $P(m) \geq \delta+1$ for some $m \in\left[b_{k}, b_{k}+\eta\right]$ with $\eta=\delta+1$. We can start in fact the induction procedure $k \mapsto k+1$ with $b_{1}=\eta+1=\delta+2$, because the only property needed for the induction step is the vanishing property

$$
H^{0}\left(X, 2 K_{X}+m L\right)=0 \quad \text { for } q \geq 1, m \geq b_{1},
$$

which is true by the Kodaira vanishing theorem and the ampleness of $K_{X}+b_{1} L$ (here we use Fujita's result (2.3), observing that $b_{1}>n+1$ ). Then the recursion formula $b_{k+1}=\left\lfloor\frac{1}{2}\left(b_{k}+\eta\right)\right\rfloor+1$ yields $b_{k}=\eta+1=\delta+2$ for all $k$, and (2.4 a) follows.

Proof of (2.4b). Quite similar to (2.4 a), except that we take $\eta=n, a=1$ and $b_{k}=n+1$ for all $k$. By Lemma $(2.2 \mathrm{~b})$, we have $P(m) \geq a_{d} k^{d} / 2^{d-1}$ for some integer $m \in\left[m_{0}, m_{0}+k d\right]$, where $a_{d}>0$ is the coefficient of highest degree in $P$. By Lemma (2.1) we have $a_{d} \geq \inf _{\operatorname{dim} Y=d} L^{d} \cdot Y$. We take $k=\lfloor n / d\rfloor$. The condition $P(m) \geq \delta+1$ can thus be realized for some $m \in\left[m_{0}, m_{0}+k d\right] \subset\left[m_{0}, m_{0}+n\right]$ as soon as

$$
\inf _{\operatorname{dim} Y=d} L^{d} \cdot Y\lfloor n / d\rfloor^{d} / 2^{d-1}>\delta
$$

which is equivalent to the condition given in $(2.4 \mathrm{~b})$.

Theorem $(0.2 \mathrm{a})$ is a special case of Th. $(2.4 \mathrm{a})$. Theorem $(0.2 \mathrm{~b})$ can be derived from $(2.4 \mathrm{~b})$ by using the following simple lemma.

(2.5) Lemma. Assume that for some integer $\mu \in \mathbb{N}^{\star}$ the line bundle $\mu F$ generates simultaneously all jets of order $\mu\left(n+s_{j}\right)+1$ at any point $x_{j}$ in a subset $\left\{x_{1}, \ldots, x_{p}\right\} \subset X$. Then $K_{X}+F$ generates simultaneously all jets of order $s_{j}$ at $x_{j}$.

Proof. Take the algebraic metric on $F$ defined by a basis of sections $\sigma_{1}, \ldots, \sigma_{N}$ of $\mu F$ which vanish at order $\mu\left(n+s_{j}\right)+1$ at all points $x_{j}$. Since we are still free to choose the homogeneous term of degree $\mu\left(n+s_{j}\right)+1$ in the Taylor expansion at $x_{j}$, we find that $x_{1}, \ldots, x_{p}$ are isolated zeroes of $\bigcap \sigma_{j}^{-1}(0)$. If $\varphi$ is the weight of the metric of $F$ near $x_{j}$, we thus have $\varphi(z) \sim\left(n+s_{j}+\frac{1}{\mu}\right) \log \left|z-x_{j}\right|$ in suitable coordinates. We replace $\varphi$ in a neighborhood of $x_{j}$ by

$$
\varphi^{\prime}(z)=\max \left(\varphi(z),|z|^{2}-C+\left(n+s_{j}\right) \log \left|z-x_{j}\right|\right)
$$

and leave $\varphi$ elsewhere unchanged (this is possible by taking $C>0$ very large). Then $\varphi^{\prime}(z)=|z|^{2}-C+\left(n+s_{j}\right) \log \left|z-x_{j}\right|$ near $x_{j}$, in particular $\varphi^{\prime}$ is strictly plurisubharmonic near $x_{j}$. In this way, we get a metric $h^{\prime}$ on $F$ with semipositive curvature everywhere on $X$, and with positive definite curvature on a neighborhood of $\left\{x_{1}, \ldots, x_{p}\right\}$. The conclusion then follows from Cor. (1.5) and Rem. (1.6).

Proof of Theorem (0.2b). By Lemma (2.5) applied with $F=K_{X}+L$ and $\mu=n+1$, the desired jet generation of $2 K_{X}+L$ occurs if $(n+1)\left(K_{X}+L\right)$ generates jets of 
order $(n+1)\left(n+s_{j}\right)+1$ at $x_{j}$. By Lemma (2.5) again with $F=a K_{X}+(n+1) L$ and $\mu=1$, we see by backward induction on $a$ that we need the simultaneous generation of jets of order $(n+1)\left(n+s_{j}\right)+1+(n+1-a)(n+1)$ at $x_{j}$. In particular, for $2 K_{X}+(n+1) L$ we need the generation of jets of order $(n+1)\left(2 n+s_{j}-1\right)+1$. Theorem $(2.4 \mathrm{~b})$ yields the desired condition.

Proof of Theorem (0.2c). Apply Th. (2.4a) with $G^{\prime}=a\left(K_{X}+(n+1) L\right)+G$, so that

$$
2 K_{X}+m L+G^{\prime}=(a+2)\left(K_{X}+(n+2) L\right)+(m-2 n-4-a) L+G,
$$

and take $m=a+2 n+4 \geq 2+\left(\begin{array}{c}3 n+1 \\ n\end{array}\right)$.

\section{An Estimate for $L^{2}$ Dualizing Sheaves}

If $Y$ is a complex $p$-dimensional analytic space with arbitrary singularities, we define the $L^{2}$ dualizing sheaf of $Y$ to be the sheaf of holomorphic $p$-forms $u$ on the regular part $Y_{\text {reg }}$ which are locally $L^{2}$ near $Y_{\text {sing }}$, that is, for any open set $W \subset Y$,

$$
\Gamma\left(W, \omega_{Y}\right)=\left\{u \in \Gamma\left(W \cap Y_{\text {reg }}, \Omega_{Y_{\text {reg }}}^{p}\right) ; \forall x \in W, \exists V \ni x, \int_{V \cap Y_{\text {reg }}} \mathrm{i}^{p^{2}} u \wedge \bar{u}<+\infty\right\},
$$

where $V$ is an arbitrary neighborhood of $x$. It is easily seen that $\omega_{Y}$ is the direct image of the dualizing sheaf $K_{\widetilde{Y}}$ of a desingularization of $Y$, thus $\omega_{Y}$ is a coherent sheaf on $Y$ ( $\omega_{Y}$ is just the usual dualizing sheaf of algebraic geometers). Then we have the following optimal "upper estimate" for $\omega_{Y}$.

(3.1) Theorem. Let $H$ be a very ample line bundle on a projective algebraic manifold $X$, and let $Y \subset X$ be a p-dimensional irreducible algebraic subvariety. If $\delta=$ $H^{p} \cdot Y$ is the degree of $Y$ with respect to $H$, the sheaf $\mathcal{H o m}\left(\omega_{Y}, \mathcal{O}_{Y}((\delta-p-2) H)\right)$ has a nontrivial section.

Observe that if $Y$ is a smooth hypersurface of degree $\delta$ in $(X, H)=\left(\mathbb{P}^{p+1}, \mathcal{O}(1)\right)$, then $\omega_{Y}=\mathcal{O}_{Y}(\delta-p-2)$ and the estimate is optimal. On the other hand, if $Y$ is a smooth complete intersection of multidegree $\left(\delta_{1}, \ldots, \delta_{r}\right)$ in $\mathbb{P}^{p+r}$, then $\delta=\delta_{1} \ldots \delta_{r}$ whilst $\omega_{Y}=\mathcal{O}_{Y}\left(\delta_{1}+\ldots+\delta_{r}-p-r-1\right)$; in this case, Th. (3.1) is thus very far from being sharp.

Proof. Let $X \subset \mathbb{P}^{N}$ be the embedding given by $H$, so that $H=\mathcal{O}_{X}(1)$. There is a linear projection $\mathbb{P}^{n}-\ldots \mathbb{P}^{p+1}$ whose restriction $\pi: Y \rightarrow \mathbb{P}^{p+1}$ to $Y$ is a finite and regular birational map of $Y$ onto an algebraic hypersurface $Y^{\prime}$ of degree $\delta$ in $\mathbb{P}^{p+1}$. Let $s \in H^{0}\left(\mathbb{P}^{p+1}, \mathcal{O}(\delta)\right)$ be the polynomial of degree $\delta$ defining $Y^{\prime}$. We claim that for any small Stein open set $W \subset \mathbb{P}^{p+1}$ and any $L^{2}$ holomorphic $p$ form $u$ on $Y^{\prime} \cap W$, there is a $L^{2}$ holomorphic $(p+1)$-form $\widetilde{u}$ on $W$ with values in $\mathcal{O}(\delta)$ such that $\widetilde{u}_{\mid Y^{\prime} \cap W}=u \wedge d s$. In fact, this is precisely the conclusion of the Ohsawa-Takegoshi extension theorem [OT87], [Ohs88] (see also [Man93] for a more 
general version); one can also invoke more standard local algebra arguments (see Hartshorne [Har77], Th. III-7.11). As $K_{\mathbb{P}^{p+1}}=\mathcal{O}(-p-2)$, the form $\widetilde{u}$ can be seen as a section of $\mathcal{O}(\delta-p-2)$ on $W$, thus the sheaf morphism $u \mapsto u \wedge d s$ extends into a global section of $\mathcal{H o m}\left(\omega_{Y^{\prime}}, \mathcal{O}_{Y^{\prime}}(\delta-p-2)\right)$. The pull-back by $\pi^{\star}$ yields a section of $\mathcal{H o m}\left(\pi^{\star} \omega_{Y^{\prime}}, \mathcal{O}_{Y}((\delta-p-2) H)\right)$. Since $\pi$ is finite and generically $1: 1$, it is easy to see that $\pi^{\star} \omega_{Y^{\prime}}=\omega_{Y}$. The Theorem follows.

\section{An Effective Version of Matsusaka's Big Theorem}

Let $L$ be an ample line bundle on a projective algebraic manifold $X$. We look for an explicit value of $m_{0}$ such that $m L$ is very ample for $m \geq m_{0}$. As in [Siu93], our starting point is the following lemma.

(4.1) Lemma. Let $F$ and $G$ be nef line bundles over $X$. If $F^{n}>n F^{n-1} \cdot G$, all large positive multiples $k(F-G), k \geq k_{0}$, have non trivial sections.

Proof. This is a special case of the holomorphic Morse inequalities (see [Dem85], [Tra95], [Siu93]). Here is a simple proof, following a suggestion of F. Catanese. We can suppose that $F$ and $G$ are very ample (otherwise, we replace $F$ and $G$ by $p F+A$ and $p G+A$ with $A$ very ample and large enough, and $p>0$ very large). Then $\mathcal{O}(k(F-G)) \simeq \mathcal{O}\left(k F-G_{1}-\ldots-G_{k}\right)$ for arbitrary members $G_{1}, \ldots, G_{k}$ in the linear system $|G|$, and the Lemma follows from Riemann-Roch by looking at the restriction morphism $H^{0}(X, \mathcal{O}(k F)) \rightarrow \bigoplus H^{0}\left(G_{j}, \mathcal{O}\left(k F_{\uparrow G_{j}}\right)\right.$.

(4.2) Corollary. Let $F$ and $G$ be nef line bundles over $X$. If $F$ is big and $m>$ $n F^{n-1} \cdot G / F^{n}$, then $\mathcal{O}(m F-G)$ can be equipped with a (possibly singular) hermitian metric $h$ with positive definite curvature form $\Theta_{h}(m F-G) \geq \varepsilon \omega, \varepsilon>0$, for some Kähler metric $\omega$.

Proof. In fact, if $A$ is ample and $\varepsilon \in \mathbb{Q}_{+}$small enough, Lemma (4.1) implies that some multiple $k(m F-G-\varepsilon A)$ has a section. Let $E$ be the divisor of this section and let $\omega=\Theta(A) \in c_{1}(A)$ be a Kähler form. Then $m F-G \equiv \varepsilon A+\frac{1}{k} E$ can be equipped with a singular metric $h$ of curvature form $\Theta_{h}(m F-G)=\varepsilon \Theta(A)+\frac{1}{k}[E] \geq \varepsilon \omega$.

We now consider the question of obtaining a nontrivial section in $m L$. The idea, more generally, is to obtain a criterion for the ampleness of $m L-B$ when $B$ is nef. In this way, one is able to subtract from $m L$ any undesirable multiple of $K_{X}$ which otherwise gets added to $L$ by the application of Nadel's vanishing theorem (for this, we simply replace $B$ by $B$ plus a multiple of $\left.K_{X}+(n+1) L\right)$.

(4.3) Proposition. Let $L$ be an ample line bundle over a projective $n$-fold $X$ and let $B$ be a nef line bundle over $X$. Then $K_{X}+m L-B$ has a nonzero section for some integer $m$ such that 


$$
m \leq n \frac{L^{n-1} \cdot B}{L^{n}}+n+1
$$

Proof. Let $m_{0}$ be the smallest integer $>n \frac{L^{n-1} \cdot B}{L^{n}}$. Then $m_{0} L-B$ can be equipped with a singular hermitian metric $h$ of positive definite curvature. By Nadel's vanishing theorem, we have

$$
H^{q}\left(X, \mathcal{O}\left(K_{X}+m L-B\right) \otimes \mathcal{I}(h)\right)=0 \quad \text { for } q \geq 1,
$$

thus $P(m)=h^{0}\left(X, \mathcal{O}\left(K_{X}+m L-B\right) \otimes \mathcal{I}(h)\right)$ is a polynomial for $m \geq m_{0}$. Since $P$ is a polynomial of degree $n$ and is not identically zero, there must be an integer $m \in\left[m_{0}, m_{0}+n\right]$ which is not a root. Hence there is a nontrivial section in

$$
H^{0}\left(X, \mathcal{O}\left(K_{X}+m L-B\right)\right) \supset H^{0}\left(X, \mathcal{O}\left(K_{X}+m L-B\right) \otimes \mathcal{I}(h)\right)
$$

for some $m \in\left[m_{0}, m_{0}+n\right]$, as desired.

(4.4) Corollary. If $L$ is ample and $B$ is nef, $m L-B$ has a nonzero section for some integer

$$
m \leq n\left(\frac{L^{n-1} \cdot B+L^{n-1} \cdot K_{X}}{L^{n}}+n+1\right) .
$$

Proof. By Fujita's result $(2.3 \mathrm{a}), K_{X}+(n+1) L$ is nef. We can thus replace $B$ by $B+K_{X}+(n+1) L$ in the result of Prop. (4.3). Corollary (4.4) follows.

(4.5) Remark. We do not know if the above Corollary is sharp, but it is certainly not far from being so. Indeed, for $B=0$, the initial constant $n$ cannot be replaced by anything smaller than $n / 2$ : take $X$ to be a product of curves $C_{j}$ of large genus $g_{j}$ and $B=0$; our bound for $L=\mathcal{O}\left(a_{1}\left[p_{1}\right]\right) \otimes \ldots \otimes \mathcal{O}\left(a_{n}\left[p_{n}\right]\right)$ to have $|m L| \neq \emptyset$ becomes $m \leq \sum\left(2 g_{j}-2\right) / a_{j}+n(n+1)$, which fails to be sharp only by a factor 2 when $a_{1}=\ldots=a_{n}=1$ and $g_{1} \gg g_{2} \gg \ldots \gg g_{n} \rightarrow+\infty$. On the other hand, the additive constant $n+1$ is already best possible when $B=0$ and $X=\mathbb{P}^{n}$.

So far, the method is not really sensitive to singularities (Lemma (4.1) is still true in the singular case as is easily seen by using a desingularization of $X$ ). The same is true with Nadel's vanishing Theorem (1.2), provided that $K_{X} \otimes \mathcal{I}(h)$ is replaced by the sheaf $\omega_{X}(h)$ of $n$-forms which are locally $L^{2}$ near $X_{\text {sing }}$ with respect to the weight $e^{-\varphi}$ of $h$ (according to that notation, the $L^{2}$ dualizing sheaf $\omega_{X}$ is associated with $\varphi=0$ or with any nonsingular weight $\varphi$ ). Then Prop. (4.3) can be generalized as

(4.6) Proposition. Let $L$ be an ample line bundle over a projective $n$-fold $X$ and let $B$ be a nef line bundle over $X$. For every p-dimensional (reduced) algebraic subvariety $Y$ of $X$, there is an integer

$$
m \leq p \frac{L^{p-1} \cdot B \cdot Y}{L^{p} \cdot Y}+p+1
$$

such that the sheaf $\omega_{Y} \otimes \mathcal{O}_{Y}(m L-B)$ has a nonzero section. 
By an appropriate induction process based on the above results, we can now improve Siu's effective version of the Big Matsusaka Theorem [Siu93]. Our version depends on a constant $\lambda_{n}$ such that $m\left(K_{X}+(n+2) L\right)+G$ is very ample for $m \geq \lambda_{n}$ and every nef line bundle $G$. Theorem (0.2 c) shows that $\lambda_{n} \leq\left(\begin{array}{c}3 n+1 \\ n\end{array}\right)-2 n$, and a similar argument involving the recent results of Angehrn-Siu [AS94] implies $\lambda_{n} \leq n^{3}-n^{2}-n-1$ for $n \geq 2$. Of course, it is expected that $\lambda_{n}=1$ in view of the Fujita conjecture.

(4.7) Effective version of the Big Matsusaka Theorem. Let $L$ and $B$ be nef line bundles on a projective $n$-fold $X$. Assume that $L$ is ample and set $H=$ $\lambda_{n}\left(K_{X}+(n+2) L\right)$. Then $m L-B$ is very ample for

$$
m \geq(2 n)^{\left(3^{n-1}-1\right) / 2} \frac{\left(L^{n-1} \cdot(B+H)\right)^{\left(3^{n-1}+1\right) / 2}\left(L^{n-1} \cdot H\right)^{3^{n-2}(n / 2-3 / 4)-1 / 4}}{\left(L^{n}\right)^{3^{n-2}(n / 2-1 / 4)+1 / 4}} .
$$

In particular $m L$ is very ample for

$$
m \geq C_{n}\left(L^{n}\right)^{3^{n-2}}\left(n+2+\frac{L^{n-1} \cdot K_{X}}{L^{n}}\right)^{3^{n-2}(n / 2+3 / 4)+1 / 4}
$$

with $C_{n}=(2 n)^{\left(3^{n-1}-1\right) / 2}\left(\lambda_{n}\right)^{3^{n-2}(n / 2+3 / 4)+1 / 4}$.

Proof. We use Th. (3.1) and Prop. (4.6) to construct inductively a sequence of (non necessarily irreducible) algebraic subvarieties $X=Y_{n} \supset Y_{n-1} \supset \ldots \supset Y_{2} \supset Y_{1}$ such that $Y_{p}=\bigcup_{j} Y_{p, j}$ is $p$-dimensional, and $Y_{p-1}$ is obtained for each $p \geq 2$ as the union of zero sets of sections

$$
\sigma_{p, j} \in H^{0}\left(Y_{p, j}, \mathcal{O}_{Y_{p, j}}\left(m_{p, j} L-B\right)\right)
$$

with suitable integers $m_{p, j} \geq 1$. We proceed by induction on decreasing values of the dimension $p$, and find inductively upper bounds $m_{p}$ for the integers $m_{p, j}$.

By Cor. (4.4), an integer $m_{n}$ for $m_{n} L-B$ to have a section $\sigma_{n}$ can be found with

$$
m_{n} \leq n \frac{L^{n-1} \cdot\left(B+K_{X}+(n+1) L\right)}{L^{n}} \leq n \frac{L^{n-1} \cdot(B+H)}{L^{n}} .
$$

Now suppose that the sections $\sigma_{n}, \ldots, \sigma_{p+1, j}$ have been constructed. Then we get inductively a $p$-cycle $\tilde{Y}_{p}=\sum \mu_{p, j} Y_{p, j}$ defined by $\widetilde{Y}_{p}=$ sum of zero divisors of sections $\sigma_{p+1, j}$ in $\widetilde{Y}_{p+1, j}$, where the mutiplicity $\mu_{p, j}$ on $Y_{p, j} \subset Y_{p+1, k}$ is obtained by multiplying the corresponding multiplicity $\mu_{p+1, k}$ with the vanishing order of $\sigma_{p+1, k}$ along $Y_{p, j}$. As cohomology classes, we find

$$
\tilde{Y}_{p} \equiv \sum\left(m_{p+1, k} L-B\right) \cdot\left(\mu_{p+1, k} Y_{p+1, k}\right) \leq m_{p+1} L \cdot \tilde{Y}_{p+1}
$$

Inductively, we thus have the numerical inequality

$$
\tilde{Y}_{p} \leq m_{p+1} \ldots m_{n} L^{n-p} .
$$

Now, for each component $Y_{p, j}$, Prop. (4.6) shows that there exists a section of $\omega_{Y_{p, j}} \otimes \mathcal{O}_{Y_{p, j}}\left(m_{p, j} L-B\right)$ for some integer 


$$
m_{p, j} \leq p \frac{L^{p-1} \cdot B \cdot Y_{p, j}}{L^{p} \cdot Y_{p, j}}+p+1 \leq p m_{p+1} \ldots m_{n} L^{n-1} \cdot B+p+1 .
$$

Here, we have used the obvious lower bound $L^{p-1} \cdot Y_{p, j} \geq 1$ (this is of course a rather weak point in the argument). The degree of $Y_{p, j}$ with respect to $H$ admits the upper bound

$$
\delta_{p, j}:=H^{p} \cdot Y_{p, j} \leq m_{p+1} \ldots m_{n} H^{p} \cdot L^{n-p} .
$$

We use the Hovanski-Teissier concavity inequality

$$
\left(L^{n-p} \cdot H^{p}\right)^{\frac{1}{p}}\left(L^{n}\right)^{1-\frac{1}{p}} \leq L^{n-1} \cdot H
$$

([Hov79], [Tei79, 82], see also [Dem93]) to express our bounds in terms of the intersection numbers $L^{n}$ and $L^{n-1} \cdot H$ only. We then get

$$
\delta_{p, j} \leq m_{p+1} \ldots m_{n} \frac{\left(L^{n-1} \cdot H\right)^{p}}{\left(L^{n}\right)^{p-1}} .
$$

By Th. (3.1), there is a nontrivial section in

$$
\mathcal{H o m}\left(\omega_{Y_{p, j}}, \mathcal{O}_{Y_{p, j}}\left(\left(\delta_{p, j}-p-2\right) H\right)\right) .
$$

Combining this section with the section in $\omega_{Y_{p, j}} \otimes \mathcal{O}_{Y_{p, j}}\left(m_{p, j} L-B\right)$ already constructed, we get a section of $\mathcal{O}_{Y_{p, j}}\left(m_{p, j} L-B+\left(\delta_{p, j}-p-2\right) H\right)$ on $Y_{p, j}$. Since we do not want $H$ to appear at this point, we replace $B$ with $B+\left(\delta_{p, j}-p-2\right) H$ and thus get a section $\sigma_{p, j}$ of $\mathcal{O}_{Y_{p, j}}\left(m_{p, j} L-B\right)$ with some integer $m_{p, j}$ such that

$$
\begin{aligned}
m_{p, j} & \leq p m_{p+1} \ldots m_{n} L^{n-1} \cdot\left(B+\left(\delta_{p, j}-p-2\right) H\right)+p+1 \\
& \leq p m_{p+1} \ldots m_{n} \delta_{p, j} L^{n-1} \cdot(B+H) \\
& \leq p\left(m_{p+1} \ldots m_{n}\right)^{2} \frac{\left(L^{n-1} \cdot H\right)^{p}}{\left(L^{n}\right)^{p-1}} L^{n-1} \cdot(B+H) .
\end{aligned}
$$

Therefore, by putting $M=n L^{n-1} \cdot(B+H)$, we get the recursion relation

$$
m_{p} \leq M \frac{\left(L^{n-1} \cdot H\right)^{p}}{\left(L^{n}\right)^{p-1}}\left(m_{p+1} \ldots m_{n}\right)^{2} \quad \text { for } 2 \leq p \leq n-1
$$

with initial value $m_{n} \leq M / L^{n}$. If we let $\left(\bar{m}_{p}\right)$ be the sequence obtained by the same recursion formula with equalities instead of inequalities, we get $m_{p} \leq \bar{m}_{p}$ with $\bar{m}_{n-1}=M^{3}\left(L^{n-1} \cdot H\right)^{n-1} /\left(L^{n}\right)^{n}$ and

$$
\bar{m}_{p}=\frac{L^{n}}{L^{n-1} \cdot H} \bar{m}_{p+1}^{2} \bar{m}_{p+1}
$$

for $2 \leq p \leq n-2$. We then find inductively

$$
m_{p} \leq \bar{m}_{p}=M^{3^{n-p}} \frac{\left(L^{n-1} \cdot H\right)^{3^{n-p-1}(n-3 / 2)+1 / 2}}{\left(L^{n}\right)^{3^{n-p-1}(n-1 / 2)+1 / 2}} .
$$

We next show that $m_{0} L-B$ is nef for

$$
m_{0}=\max \left(m_{2}, m_{3}, \ldots, m_{n}, m_{2} \ldots m_{n} L^{n-1} \cdot B\right) .
$$


In fact, let $C \subset X$ be an arbitrary irreducible curve. Either $C=Y_{1, j}$ for some $j$ or there exists an integer $p=2, \ldots, n$ such that $C$ is contained in $Y_{p}$ but not in $Y_{p-1}$. If $C \subset Y_{p, j} \backslash Y_{p-1}$, then $\sigma_{p, j}$ does not vanish identically on $C$. Hence $\left(m_{p, j} L-B\right)_{\uparrow C}$ has nonnegative degree and

$$
\left(m_{0} L-B\right) \cdot C \geq\left(m_{p, j} L-B\right) \cdot C \geq 0 .
$$

On the other hand, if $C=Y_{1, j}$, then

$$
\left(m_{0} L-B\right) \cdot C \geq m_{0}-B \cdot \widetilde{Y}_{1} \geq m_{0}-m_{2} \ldots m_{n} L^{n-1} \cdot B \geq 0 .
$$

By the definition of $\lambda_{n}$ (and the proof of (0.2 c) that such a constant exists), $H+G$ is very ample for every nef line bundle $G$, in particular $H+m_{0} L-B$ is very ample. We thus replace again $B$ with $B+H$. This has the effect of replacing $M$ with $M=n\left(L^{n-1} \cdot(B+2 H)\right)$ and $m_{0}$ with

$$
m_{0}=\max \left(m_{n}, m_{n-1}, \ldots, m_{2}, m_{2} \ldots m_{n} L^{n-1} \cdot(B+H)\right) .
$$

The last term is the largest one, and from the estimate on $\bar{m}_{p}$, we get

$$
\begin{aligned}
m_{0} & \leq M^{\left(3^{n-1}-1\right) / 2} \frac{\left(L^{n-1} \cdot H\right)^{\left(3^{n-2}-1\right)(n-3 / 2) / 2+(n-2) / 2}\left(L^{n-1} \cdot(B+H)\right)}{\left(L^{n}\right)^{\left(3^{n-2}-1\right)(n-1 / 2) / 2+(n-2) / 2+1}} \\
& \leq(2 n)^{\left(3^{n-1}-1\right) / 2} \frac{\left(L^{n-1} \cdot(B+H)\right)^{\left(3^{n-1}+1\right) / 2}\left(L^{n-1} \cdot H\right)^{3^{n-2}(n / 2-3 / 4)-1 / 4}}{\left(L^{n}\right)^{3^{n-2}(n / 2-1 / 4)+1 / 4}}
\end{aligned}
$$

(4.8) Remark. In the surface case $n=2$, one can take $\lambda_{n}=1$ and our bound yields $m L$ very ample for

$$
m \geq 4 \frac{\left(L \cdot\left(K_{X}+4 L\right)\right)^{2}}{L^{2}}
$$

If one looks more carefully at the proof, the initial constant 4 can be replaced by 2 . In fact, it has been shown recently by Fernández del Busto that $m L$ is very ample for

$$
m>\frac{1}{2}\left[\frac{\left(L \cdot\left(K_{X}+4 L\right)+1\right)^{2}}{L^{2}}+3\right]
$$

and an example of G. Xiao shows that this bound is essentially optimal (see [FdB94]).

\section{References}

[AN54] AkizUki, Y., NAKAnO, S. - Note on Kodaira-Spencer's proof of Lefschetz theorems, Proc. Jap. Acad., 30 (1954), 266-272.

[AV65] Andreotti, A., Vesentini, E. - Carleman estimates for the Laplace-Beltrami equation in complex manifolds, Publ. Math. I.H.E.S., 25 (1965), 81-130.

[AS94] Angehrn, U., SiU, Y.T. - Effective freeness and point separation for adjoint bundles, Preprint December 1994. 
[Dem85] Demailly, J.-P. - Champs magnétiques et inégalités de Morse pour la d" -cohomologie, Ann. Inst. Fourier (Grenoble), 35 (1985), 189-229.

[Dem90] Demailly, J.-P. - Singular hermitian metrics on positive line bundles, Proc. Conf. Complex algebraic varieties (Bayreuth, April 2-6, 1990), edited by K. Hulek, T. Peternell, M. Schneider, F. Schreyer, Lecture Notes in Math., Vol. 1507, SpringerVerlag, Berlin (1992).

[Dem93] Demailly, J.-P. - A numerical criterion for very ample line bundles, J. Differential Geom., 37 (1993), 323-374.

[Ein94] EIN, L. - Note on higher dimensional adjoint linear systems, Preprint 1994, personal communication to the author.

[EKL94] Ein, L., KüCHLE, O., LAzARsfeld, R. - Local positivity of ample line bundles, Preprint August 1994, to appear.

[EL92] EIN, L., LAZARSFELD, R. - Seshadri constants on smooth surfaces, Journées de Géométrie Algébrique d'Orsay, July 1992, Astérisque, 282 (1993), 177-186.

[EL93] EIN, L., LAZARSFELD, R. - Global generation of pluricanonical and adjoint linear series on smooth projective threefolds, Jour. of Am. Math. Soc., 6 (1993), 875-903.

[FdB94] Fernández Del Busto, G. - A Matsusaka-type theorem for surfaces, Preprint Princeton University, July 1994.

[Fuj87] FUJITA, T. - On polarized manifolds whose adjoint bundles are not semipositive, Algebraic Geometry, Sendai, 1985, Adv. Stud. in Pure Math., North Holland, T. Oda (ed.), 10 (1987), 167-178.

[Fuj94] FuJITA, T. - Remarks on Ein-Lazarsfeld criterion of spannedness of adjoint bundles of polarized threefolds, Preprint 1994, to appear.

[Har77] HARTSHORNE, R. - Algebraic geometry, Springer-Verlag, Berlin, 1977.

[Hör65] HÖRMANDER, L. - $L^{2}$ estimates and existence theorems for the $\bar{\partial}$ operator, Acta Math., 113 (1965), 89-152.

[Hov79] Hovanski, A.G. - Geometry of convex bodies and algebraic geometry, Uspehi Mat. Nauk, 34 (1979), 160-161.

[Kaw82] KawAmATA, Y. - A generalization of Kodaira-Ramanujam's vanishing theorem, Math. Ann., 261 (1982), 43-46.

[Kaw84] KaWAmATA, Y. - The cone of curves of algebraic varieties, Ann. of Math., 119 (1984), 603-633.

[Kaw85] KaWAMATA, Y. - Pluricanonical systems of minimal algebraic varieties, Invent. Math., 79 (1985), 567-588.

[Kol93] KollÁR, J. - Effective basepoint freeness, Math. Ann., 296 (1993), 595-605.

[KoM83] Kollár, J., MatsusakA, T. - Riemann-Roch type inequalities, Amer. J. of Math., 105 (1983), 229-252.

[Laz93] LAZARsfeld, R., with the assistance of Fernández DEL Busto, G. Lectures on linear series, Park City, IAS Mathematics Series, Vol. 3 (1993).

[Man93] MANIVEL, L. _ Un théorème de prolongement $L^{2}$ de sections holomorphes d'un fibré vectoriel, Math. Zeitschrift, 212 (1993), 107-122.

[Mat72] MatsusAKA, T. - Polarized varieties with a given Hilbert polynomial, Amer. J. of Math., 94 (1972), 1027-1077.

[Mor82] MORI, S. - Threefolds whose canonical bundles are not numerically effective, Ann. of Math., 116 (1982), 133-176.

[Nad89] NADEL, A.M. - Multiplier ideal sheaves and Kähler-Einstein metrics of positive scalar curvature, Proc. Nat. Acad. Sci. U.S.A., 86 (1989), 7299-7300 and Ann. of Math. 132 (1990), 549-596.

[Nak55] NAKANO, S. - On complex analytic vector bundles, J. Math. Soc. Japan., 7 (1955), $1-12$.

[Ohs88] OHSAWA, T. - On the extension of $L^{2}$ holomorphic functions, II, Publ. RIMS, Kyoto Univ., 24 (1988), 265-275.

[OT87] OHSAWA, T., TAKEGOSHI, K. - On the extension of $L^{2}$ holomorphic functions, Math. Zeitschrift, 195 (1987), 197-204.

[Rei88] REIDER, I. - Vector bundles of rank 2 and linear systems on algebraic surfaces, Ann. of Math., 127 (1988), 309-316. 
[Siu93] SIU, Y.T. — An effective Matsusaka big theorem, Ann. Inst. Fourier., 43 (1993), 1387-1405.

[Siu94a] SiU, Y.T. - Effective Very Ampleness, Preprint 1994, to appear in Inventiones Math.

[Siu94b] SIU, Y.T. - Very ampleness criterion of double adjoint of ample line bundles, Preprint 1994, to appear in Annals of Math. Studies, volume in honor of Gunning and Kohn, Princeton Univ. Press.

[Tei79] TEISSIER, B. - Du théorème de l'index de Hodge aux inégalités isopérimétriques, C. R. Acad. Sc. Paris, Sér. A,, 288 (1979), 287-289.

[Tei82] TEISSIER, B. - Bonnesen-type inequalities in algebraic geometry, Sem. on Diff. Geom. edited by S.T. Yau, 1982, Princeton Univ. Press, 85-105.

[Tra95] TRAPANI, S. - Numerical criteria for the positivity of the difference of ample divisors, Math. Zeitschrift, 219 (1995), 387-401.

[Vie82] VieHWEG, E. - Vanishing theorems, J. Reine Angew. Math., 335 (1982), 1-8.

Jean-Pierre Demailly

Université de Grenoble I, Institut Fourier, BP74

F-38400 Saint-Martin d'Hères, France

e-mail: demailly@fourier.ujf-grenoble.fr 